\title{
Audit of combination and high-dose antipsychotic treatment in the community
}

\author{
Lovely Rajan, ${ }^{1}$ Ian Clarke $^{1}$
}

The Psychiatrist (2013), 37, 302-307, doi: 10.1192/pb.bp.112.039750

${ }^{1} \mathrm{NHS}$ Greater Glasgow \& Clyde, Glasgow, Scotland

Correspondence to Lovely Rajan (lovely.rajan@nhs.net)

First received 23 Apr 2012, final revision 25 Feb 2013, accepted 4 Apr 2013

\begin{abstract}
Aims and method To estimate the community prevalence of combination antipsychotic therapy and high-dose antipsychotic treatment via audit. Patients with an ICD-10 diagnosis of schizophrenia were identified through the NHS Greater Glasgow and Clyde Health Board patient information management system and their mental health records examined.
\end{abstract}

Results In audit 1, 135 records were examined. The age range of patients was 24-74 years; $73 \%$ were male. Twelve per cent were on combined antipsychotic treatment and $3.7 \%$ required high-dose antipsychotics. Sixty-nine per cent of patients had documented in their record the reason for combined antipsychotic treatment compared with $40 \%$ on high-dose antipsychotics. In audit 2 (1 year later), 150 records were examined. Demographic details and prescription patterns remained similar to that of audit 1 . However, $10 \%$ of patients received combined antipsychotic therapy and $2 \%$ remained on high-dose antipsychotics. Documented reasons for combination therapy increased to $87 \%$, and to $67 \%$ for patients on high-dose antipsychotics.

Clinical implications We need robust clinical trials, overcoming the problems of clinical effectiveness and clinical efficiency, to establish the merits of preferred antipsychotic combinations, as combination and high-dose antipsychotic treatments are unlikely to be eliminated in clinical practice in the foreseeable future.

Declaration of interest L.R. was a specialty doctor at Arndale Mental Health Resource Centre at the time of the study and I.C. is one of two consultants based at Arndale Mental Health Resource Centre.

\begin{abstract}
Many people with psychotic illnesses respond poorly to the medication regimens in published guidelines which, however worthy, are often of limited help in 'real world settings'. This is perhaps one reason why combination antipsychotic therapy and/or high-dose antipsychotic treatment are common in clinical practice despite published recommendations and lack of research evidence. The Royal College of Psychiatrists' consensus statement on high-dose antipsychotic medication defines it as 'a total daily dose of a single antipsychotic which exceeds the upper limit stated in the British National Formulary (BNF) [...] or a total daily dose of two or more antipsychotics which exceeds the BNF maximum using the percentage method'. ${ }^{1}$ Similarly, combination antipsychotic therapy is defined as 'the concurrent use of two or more antipsychotics. ${ }^{1}$

There are published reports of combination and highdose antipsychotic treatment from a number of countries. For example, an audit of $>3000$ psychiatric in-patients in the UK found that $48 \%$ of patients were on combined antipsychotic therapy and $20 \%$ on high-dose antipsychotics, ${ }^{2}$ and an Australian study of out-patients with schizophrenia showed a $13 \%$ rate of combination antipsychotic therapy. ${ }^{3}$ Japanese studies reported that combined antipsychotics were used in $>90 \%$ of patients ${ }^{4}$ and a prevalence of $69 \%$ in
\end{abstract}

$2005 .{ }^{5}$ Studies of clinical practice in Scotland found a prevalence of $30 \%$ for combined antipsychotic treatment, ${ }^{6}$ whereas a report from Wales found prevalence rates of $67.3 \%$ for combined therapy and $13.7 \%$ for high-dose antipsychotic treatment. ${ }^{7}$ More recently, a study from London, UK, reported prevalence rates of $10 \%$ and $55 \%$ for combined and high-dose antipsychotic treatment respectively. ${ }^{8}$

Although combined antipsychotics may increase adverse drug effects, some have argued that certain antipsychotic combinations may have benefits; ${ }^{8,9}$ for example, adding aripiprazole to clozapine may help some patients to lose weight and improve metabolic abnormalities. ${ }^{10}$ Similarly, co-therapy with aripiprazole and haloperidol has been reported to help normalise prolactin levels in patients treated with haloperidol alone. ${ }^{\text {ll }}$ One polypharmacy approach commonly used is clozapine augmentation which has been reported to improve positive and negative symptoms. ${ }^{12-14}$

\section{Guidance}

To promote good practice of combination/high-dose antipsychotic treatment, a number of guidelines have been 
published. In the UK, the National Institute for Health and Care Excellence (NICE) states 'do not initiate regular combined antipsychotic medication, except for short periods (for example, when changing medication), ${ }^{15}$ and BNF guidance ${ }^{16}$ does not recommend prescribing more than one antipsychotic at the same time. The Royal College of Psychiatrists' consensus statement comments that exceeding the recommended antipsychotic dose range is likely to exceed the acceptable risk-benefit ratio, , and the Maudsley Prescribing Guidelines recommend that 'the use of high-dose antipsychotics should be an exceptional clinical practice and only ever employed when standard treatments, including clozapine, have failed'. ${ }^{17}$

On the back of these national guidelines, local policies have been developed throughout the UK; for example, in Glasgow the high-dose antipsychotic therapy guidelines recommend that 'combination antipsychotic therapy should only be used as part of a considered treatment plan'. ${ }^{18}$ Given these treatment recommendations, we were interested in the question: how does actual prescribing practice in a community setting compare with these treatment recommendations?

The aim of the present study was to assess prescribing practice for a sample of patients with psychotic illness treated by a community mental health team based in West Glasgow (Arndale Mental Health Resource Centre) and compare this with published guidelines and findings from other centres.

\section{Method}

The Arndale Mental Health Resource Centre is located in Drumchapel, in West Glasgow. The catchment area of the resource centre encompasses a population from a wide spectrum of Scottish Index of Multiple Deprivation (SIMD). The centre has a case-load of about 700 adults with mental health problems. Gartnavel Royal Hospital provides in-patient care for patients from this catchment. Patients attending the Arndale Mental Health Resource Centre with ICD-10 ${ }^{19}$ F20-F29 diagnoses were identified using the NHS Greater Glasgow and Clyde Health Board patient information management system (PiMS). Information on demographics, diagnosis, age at onset, duration of illness, treatment prescribed, rationale for combination/high-dose antipsychotic treatment was collected for this cohort from case notes, prescription sheets and staff. We recorded details of antipsychotic prescription, route of administration, daily dose, combination treatment and high-dose prescription, documentation in relation to rationale and informed consent for combined/high-dose treatment, and information about recommended monitoring of these patients.

We defined combined antipsychotic therapy (CAT) operationally as the concurrent use of $>1$ antipsychotic for $>3$ months and high-dose antipsychotic treatment (HDAT) $>100 \%$ BNF daily dose, using aggregate percentages. ${ }^{18,20,21}$ The main audit standards were derived from existing guidelines: $:^{1,15,18}$

1 combination antipsychotic treatment should be no more than $10 \%$ of the total of patients receiving antipsychotics
2 high-dose antipsychotic treatment should be no more than $5 \%$ of the total of patients receiving antipsychotics

3 all patients on combined/high-dose antipsychotic treatment should have information in their health record regarding rationale and consent for this treatment

4 all patients receiving high-dose antipsychotics should be identified and monitored as per recommended guidance.

Results of the first audit cycle (audit 1) with recommendations were presented in a structured format using a PowerPoint presentation to multidisciplinary staff at the Arndale Mental Health Resource Centre (including medical staff members) as part of our monthly lunch-time team teaching session to increase staff awareness. Patients on combined/high-dose antipsychotic treatment were subsequently identified using their unique PiMS number and the nurse team leader was responsible for prioritising these patients for an early multidisciplinary review using an integrated care pathway. ${ }^{22}$ Results were also presented in the same format at Gartnavel Royal Hospital as part of the internal teaching programme and as a poster at the NHS Scotland Event in Glasgow. The audit was repeated after 1 year (audit 2), gathering similar information as in the first cycle to identify any change in practice. The results of the completed audit were presented at different venues as part of advanced trainees' teaching in Glasgow, the National Audit Conference in Edinburgh and as a poster at the 2012 Royal College of Psychiatrists' International Congress in Liverpool.

\section{Statistical analysis}

There were 135 patients in audit 1 and 150 patients in audit 2 (135 patients from audit 1 plus an additional 15 patients). For statistical analysis, these data cannot be considered as two independent samples. One approach in such a situation would be to impute the missing data (e.g. when baseline values in a clinical trial are carried forward for missing follow-up data). However, the data in this study were missing from the first and not the second audit, so there is not a logical way to impute this. The only statistical approach would therefore be to use paired methods of analysis and to compare categorical data using McNemar's chi-squared test.

Results are presented for each audit descriptively for comparison purposes. Since 135 of the patients were followed up, statistical analysis was done using McNemar's chi-squared test, a non-parametric test used in paired samples after removing the results for the additional 15 patients in audit 2 .

\section{Results}

\section{Audit 1}

A total of 150 patients meeting entry criteria were identified; 8 were excluded $(n=4$ F33 diagnosis; $n=3$ transferred elsewhere; $n=1$ deceased). Out of 142 cases, information was gathered on 135 patients; $72.6 \%$ were male and $27.4 \%$ were female ( 7 case notes were unavailable at the 
time of audit). Patients' age ranged from 24 to 74 years (mean 46 years) in the HDAT group compared with 29-70 years (mean 45 years) in the CAT group. Mean duration of illness was 18 years (range 1-48) in the HDAT group and 20 years (range 2-38) in the CAT group. At the time of the study, $98.5 \%$ of the patients lived in the community and $1.5 \%$ were in-patients. Most patients had a diagnosis of schizophrenia (ICD-10 code F20, $n=94$ ): other diagnoses included schizoaffective disorder (F25, $n=21$ ), persistent delusional disorders (F22, $n=10$ ), schizotypal disorder (F21, $n=7$ ), and acute and transient psychotic disorders (F23, $n=3)$.

Table 1 shows that $88 \%(n=118 / 135)$ of the population studied received high-dose antipsychotics and $12 \%(n=16 /$ 135) were on combination antipsychotic treatment; this is $2 \%$ in excess of the standard set at the onset. Of the total sample, $3.7 \% \quad(n=5 / 135) \quad$ (but $31 \% \quad(n=5 / 16)$ receiving combined antipsychotics) received high-dose antipsychotic treatment, meeting the standard originally set. Ten per cent of the total sample had $100 \%$ BNF daily dose (most of these patients were on olanzapine $20 \mathrm{mg}$ /day). Of the CAT group, $38 \%$ were prescribed an anticholinergic, as opposed to $21 \%$ of the HDAT group. Eighty per cent of the total sample received oral medication (Table 2), $32 \%$ were on depot medication (Table 3) and $12 \%$ were on combined antipsychotic therapy. The most common combination was a depot antipsychotic with oral chlorpromazine or haloperidol. The most frequently used drug was clozapine (22\%), followed by amisulpride (15\%) and olanzapine (14\%). The preferred drug for high-dose therapy was clozapine (21\%) and for combination treatment, amisulpride $(50 \%)$.

The rationale for combined antipsychotic therapy was described in $69 \%(11 / 16)$ of patients; the most common reason was to improve symptom control. In the remaining five cases, there was no recorded evidence of discussion about combination treatment. Forty per cent $(2 / 5)$ of patients on high-dose antipsychotics had recorded evidence of monitoring in relation to blood tests, electrocardiogram (ECG) and other physical health checks.

Practice recommendations after audit 1 were the following:

- regular updating of information, including transfer, discharge and change in ICD-10 diagnosis

- clear recording of current antipsychotic drug treatment with reason for, and duration of, high-dose/combined antipsychotic treatment

- in patients on combination antipsychotic treatment, regular review to justify its use; reduce if possible

- in patients on high-dose antipsychotics, regular review to justify its use; reduce if possible

- review and improve monitoring of patients on combined/ high-dose antipsychotics using an integrated care pathway to facilitate this process. ${ }^{22}$

\section{Audit 2}

In total, 156 patients were identified; 6 individuals were excluded ( $n=1$ F33 diagnosis; $n=5$ discharged or transferred to other areas), resulting in 150 patients included in audit 2 . Of the sample, $75 \%$ was male and $25 \%$ was female. The age range for the HDAT group was 24-76 years (mean 48 years) compared with 33-71 years (mean 46 years) in the CAT group. In this audit, $98 \%$ of patients lived in the community and $2 \%$ were in-patients.

In this cycle, $10 \%(n=15 / 150)$ of patients received combined antipsychotic therapy compared with $12 \% \quad(n=16 /$ 135) in audit 1 (McNemar's $P=0.25$ for $12 \% v$. $9.6 \%$ on $n=135$ paired samples). Two per cent $(n=3 / 150)$ of the sample received high-dose antipsychotics in comparison to $3.7 \%(n=5 / 135)$ in audit 1 (McNemar's $P=0.50$ for $3.7 \% v$. $2.2 \%$ on $n=135$ paired samples). Among the additional 15 patients in audit 2,2 patients were on combined antipsychotic treatment and none received high-dose antipsychotics. In $87 \%(n=13 / 15)$ of the CAT group, there was better recording of rationale, discussion with the patient, medication update and monitoring of blood tests; this was achieved by giving priority to reviewing patients on combined/high-dose antipsychotics using an integrated care pathway. ${ }^{22}$

Prescription patterns of antipsychotic medication remained much the same and, again, clozapine was the most commonly prescribed medication, followed by amisulpride and olanzapine. Prescription of aripiprazole had increased from 3 to 7 patients and that of chlorpromazine was reduced by $30 \%$, from 11 to 8 patients.

We would argue that removing three patients from the CAT group and two patients from the HDAT group is clinically significant as combination therapy is the main reason for high-dose antipsychotic treatment, and the latter can cause serious harm to patients, including cardiac toxicity and sudden death. This was achieved by targeted enhanced review and monitoring of patients on combined and high-dose antipsychotics using an integrated care pathway. We looked for differences in the recording of patients' monitoring when receiving any antipsychotic treatment in both audits. The results were $1.5 \%(n=2 / 135)$ in audit 1 and $10.6 \%(n=16 / 150)$ in audit 2 (McNemar's $P<0.001$ for $1.5 \%$ v. $12.0 \%$ on $n=135$ paired samples). In audit $1,40 \%(n=2 / 5)$ of the HDAT group were monitored and in audit $2,67 \%(n=2 / 3)$ were monitored.

Clozapine was the favoured antipsychotic medication for high-dose antipsychotic treatment, followed by amisulpride and olanzapine in both audits, consistent with other reports of the use of clozapine in chronic and treatment-resistant cases of schizophrenia. ${ }^{14,15,22,23}$ Prescription of aripiprazole increased by more than double in 1 year and was more commonly used in patients who were mentally stable but experienced side-effects, especially weight gain. It was occasionally used as an adjunct to clozapine, as evidence suggests helping patients to lose weight and to improve other metabolic parameters. ${ }^{14}$ The prevalence of chlorpromazine or haloperidol prescription, in addition to depot medication, for agitation or insomnia fell by $30 \%$ in audit 2 .

\section{Discussion}

Several audits over the past two decades have highlighted the growing prevalence of combination and high-dose antipsychotic treatment and the significant variation of recommended practice has been noted by various authors. $^{2,5-9,14,21-25}$ However, there have been few 


\begin{tabular}{lccc} 
Table 1 & Number of patients receiving high-dose and combination antipsychotics & $\begin{array}{c}\text { Patients receiving one } \\
\text { antipsychotics, \% }(n)(n=16)\end{array}$ & $\begin{array}{c}\text { Total patients, } \\
(n=135)\end{array}$ \\
$\begin{array}{l}\text { British National Formulary } \\
\text { dose, } \%\end{array}$ & $89(105)$ & $69(11)$ & $87(116)$ \\
\hline$<100$ & $11(13)$ & $0(0)$ & $10(13)$ \\
\hline 100 & $0(0)$ & $31(5)$ & $3.7(5)$ \\
\hline$>100$ & & &
\end{tabular}

a. One patient was taking no medication.

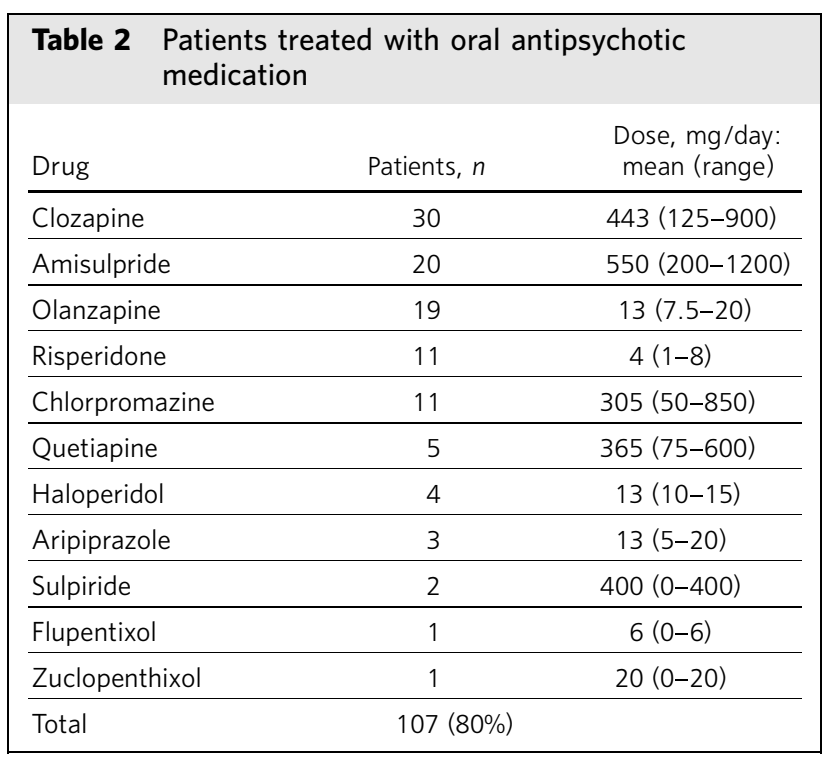

local guidelines for patients with schizophrenia who fall into this subgroup but who have difficulties with medication adherence and/or refuse blood monitoring.

Compared with the results of other published audits, including one community-based study, ${ }^{7}$ our results show relatively lower prevalence rates of combination and high-dose antipsychotic treatment in both audit cycles. However, despite the widespread use of second-generation antipsychotics, there has been no reduction in the use of combination or high-dose antipsychotic treatment. This is perhaps not surprising given that first- and second-generation antipsychotics have similar efficacy. ${ }^{26}$

Schizophrenia is associated with increased mortality which is not explained by an increased suicide rate alone. ${ }^{14,22,27}$ Patients with schizophrenia are at higher risk of cardiovascular morbidity and mortality and the development of diabetes mellitus and metabolic syndrome. ${ }^{14,28}$ In this audit, physical health monitoring of patients prescribed with combination and high-dose antipsychotics improved from $40 \%$ to $67 \%$, but this is still not satisfactory as many patients did not receive, or did not have any record of having received, monitoring as per published recommendations. If levels of high-dose antipsychotics and combination antipsychotics are higher than detected in this audit, due to discretionary self-medication, ${ }^{29}$ then it is likely that many more patients will require physical health monitoring. An integrated care pathway could be a beneficial tool in patient monitoring. ${ }^{22}$ An integrated care pathway is a multidisciplinary outline of anticipated care, placed in an appropriate time frame to help a patient with a specific condition (e.g. schizophrenia, depression, dementia) move progressively through a clinical experience to positive outcomes. Such a care pathway incorporates local and national guidelines into everyday practice, to manage clinical risk and meet the requirements of clinical governance.

The NICE update on the treatment of schizophrenia recommends that general practitioners should take

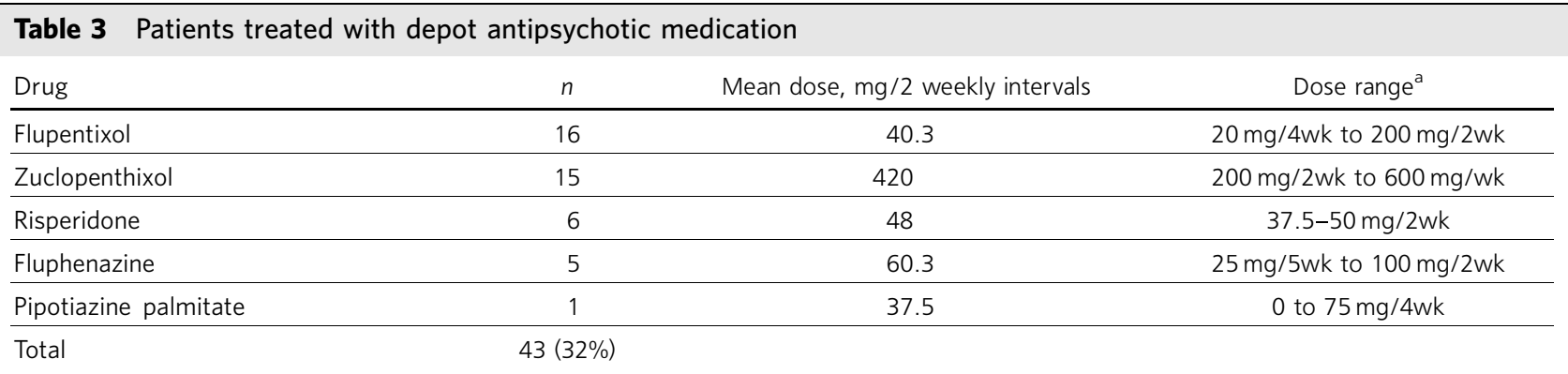

a. $n w k=$ number of weekly intervals. 
responsibility for organising physical health monitoring, including ECG and blood tests, and to share this information with psychiatrists for better patient care. ${ }^{15,22}$ How the physical health of mentally ill populations will be monitored and delivered is an important topic for future research.

The prevalence of combination antipsychotic therapy reduced from $12 \%$ to $10 \%$ in 1 year between the two audit cycles. Similarly, the prevalence of high-dose antipsychotic treatment reduced from $3.7 \%$ (5 patients) to $2 \%$ (3 patients) in 1 year at re-audit. Patients who remained on high-dose antipsychotics at re-audit had treatment-resistant schizophrenia and an increased risk of violence and aggression. This subset of patients will always present therapeutic challenges and one could argue that guidelines developed for the majority of patients with schizophrenia are probably not appropriate and that more targeted guidance is required. This would of course be in keeping with current notions of a more personalised or 'stratified' treatment approach. ${ }^{9}$

Although male patients were represented in both the HDAT and CAT groups, there were more males in the HDAT group than in the CAT group $(74 \% v .62 \%)$. This was reversed for female patients, with a relatively higher proportion of females in the CAT group than in the HDAT group (38\% v. 26\%). We could not explain the reason for this and recommend future research on this area.

As in previous studies, we found lack of compliance with available guidelines which may reflect difficulties in applying them in practice. Various difficulties in terms of staff time, resources, patients' adherence to treatment and problems obtaining a second opinion were all reported as hindering compliance with guidelines. ${ }^{8,23,25}$

\section{Limitations of the audit}

This was a retrospective study that used information available through PiMS and case notes. We did not look for details of use of the Mental Health Act 1983 or ethnicity (we had only a handful of patients treated under a compulsory treatment order and the population studied was predominantly White Scottish), and we were unable to obtain information on the concomitant use of other psychotropic medication, including discretionary antipsychotics and benzodiazepines, owing to a lack of clarity about daily dose and frequency of use of 'as required' medication. Patients in the community are reviewed less often and given that the use of 'as required' psychotropic medication is within the patient's control, the dose is likely to vary from day to day depending on the mental well-being of individual patients. This is an important consideration and suggests that levels of combination and high-dose antipsychotic treatment are likely to be higher in practice than reported. This could be an important focus for future research.

\section{Future recommendations}

Regular audits within community services should be undertaken to drive improvements in practice as recommended by NICE guidance. National Health Service organisations have a responsibility to monitor adherence to combination and high-dose antipsychotic treatment monitoring protocols as part of clinical governance and cases of serious side-effects or mortality could give rise to litigation. Targeted, evidence-based prescribing using, where applicable, non-pharmacological interventions, may be the way forward to ensure patient safety and to improve quality of life in patients with a devastating and life-long condition such as schizophrenia.

\section{Acknowledgements}

We thank Professor Robert Hunter, consultant psychiatrist, Rehabilitation Unit, Gartnavel Royal Hospital and Clinical Director of the Psychiatric Research Institute of Neuroscience, PsyRING, NHS Greater Glasgow and Clyde Health Board, for constructive criticism and contribution in writing up this project, and Mrs Emma Banks for administrative support. We also thank Dr Rajeev Krishnadas, clinical lecturer, University of Glasgow and Sackler Institute of Psychobiological Research, Glasgow, and Dr David Young, senior lecturer, Department of Mathematics and Statistics, University of Strathclyde, Glasgow, for the statistical analysis of the data. We extend our thanks to all staff at the Arndale Mental Health Resource Centre for their encouragement and involvement, particularly Mrs Tina Cuthill for providing all necessary information.

\section{About the authors}

Lovely Rajan, Advanced Trainee (ST6) in General Adult Psychiatry, Brand Street Resource Centre and Southern General Hospital, NHS Greater Glasgow and Clyde Health Board, Glasgow, Scotland. Ian Clarke, Lead Clinician and Consultant Psychiatrist, Arndale Mental Health Resource Centre and Gartnavel Royal Hospital, NHS Greater Glasgow and Clyde Health Board, Glasgow, Scotland.

\section{References}

1 Royal College of Psychiatrists. Consensus Statement on High-Dose Antipsychotic Medication (Council Report CR138). Royal College of Psychiatrists, 2006

2 Harrington M, Lelliott C, Paton C, Okocha C, Duffett R, Sensky T. The results of a multi-centre audit of the prescribing of antipsychotic drugs for in-patients in the UK. Psychiatr Bull 2002; 26: 414-8.

3 Keks NA, Alston K, Hope J, Krapivensky N, Culhane C, Tanaghow A et al. Use of antipsychotics and adjunctive medications by inner urban community psychiatric service. Aust N Z J Psychiatry 1999; 33: 896-901.

4 Ito C, Kubota Y, Sato M. A prospective survey on drug choice for prescriptions for admitted patients with schizophrenia. Psychiatry Clin Neurosci 1999; 53 (suppl): S35-40.

5 Ito H, Koyama A, Higuchi T. Polypharmacy and excessive dosing: psychiatrists' perceptions of antipsychotic drug prescription. $\mathrm{Br} J$ Psychiatry 2005; 187: 243-7.

6 Taylor M, Shajahan P, Lawrie SM. Comparing the use and discontinuation of antipsychotics in clinical practice: an observational study. J Clin Psychiatry 2008; 69: 240-5.

7 Tungaraza TE, Gupta S, Jones J, Poole R, Slegg G. Polypharmacy and high-dose antipsychotic regimes in the community. Psychiatrist 2010; 34: 44-6.

8 Grech P, Taylor D. Long-term antipsychotic polypharmacy: how does it start, why does it continue? Therapeutic Advances in Psychopharmacology 2012; 2: 5-11.

9 Taylor D. Antipsychotic polypharmacy - confusion reigns. Psychiatrist 2010; 34: 41-3.

10 Fleischhacker WW, Heikkinen $T$, Olie JP, Landsberg W, Dwaele $\mathrm{P}$, McQuade RD, et al. Weight change on aripiprazole-clozapine combination in schizophrenic patients with weight gain and 
suboptimal response on clozapine: 16-week double-blind study. Eur Psychiatry 2008; 2 (suppl 23): s114-5.

11 Shim JC, Shin JG, Kelly DL, Jung DU, Seo YS, Liu KH et al. Adjunctive treatment with a dopamine partial agonist, aripiprazole, for antipsychotic-induced hyperprolactinemia: a placebo controlled trail. Am J Psychiatry 2007; 164: 1404-10.

12 Josiassen RC, Joseph A, Kohegyi E, Stokes S, Dadvand M, Paing WW, et al. Clozapine augmented with risperidone in the treatment of schizophrenia: a randomised, double-blind, placebo-controlled trial. Am J Psychiatry 2005; 162: 130-6.

13 Shiloh R, Zemishlany Z, Aizenberg D, Radwan M, Schwartz B, Dorfman-Etrog $P$, et al. Sulpiride augmentation in people with schizophrenia partially responsive to clozapine. A double-blind placebo-controlled study. Br J Psychiatry 1997; 171: 569-73.

14 Langan J, Shajahan P. Antipsychotic polypharmacy: review of mechanisms, mortality and management. Psychiatrist 2010; 34: 58-62.

15 National Collaborating Centre for Mental Health. Schizophrenia: The NICE Guideline on Core Interventions in the Treatment and Management of Schizophrenia in Adults in Primary and Secondary Care (Updated Edition). British Psychological Society and Royal College of Psychiatrists, 2010.

16 Royal Pharmaceutical Society of Great Britain, British Medical Association. British National Formulary 56. BMJ Books \& Pharmaceuticals Press, 2008.

17 Taylor D, Paton C, Kapur S. The Maudsley Prescribing Guidelines (10th edn). Informa Healthcare, 2009.

18 NHS Greater Glasgow and Clyde Health Board. High Dose Antipsychotic Therapy Guideline. NHS Greater Glasgow and Clyde, 2008.

19 World Health Organization. The ICD-10 Classification of Mental and Behavioural Disorders: Clinical Descriptions and Diagnostic Guidelines. WHO, 1992
20 Yorston G, Pinney A. Use of high dose antipsychotic medication. Psychiatr Bull 1997; 21: 566-9.

21 Hayhurst K. Pennine Care NHS Trust Antipsychotic Prescribing Audit. The University of Manchester, 2006 (http://www.south.manchester.ac.uk/ psychiatry/capri/documents/PennineCareAntipsychoticPrescribing Audit2005.pdf)

22 NHS Quality Improvement Scotland. Standards for Integrated Care Pathways for Mental Health. NHS Quality Improvement Scotland, 2007.

23 Howes OD, Vergunst F, Gee S, McGuire P, Kapur S, Taylor D. Adherence to treatment guidelines in clinical practice: study of antipsychotic treatment prior to clozapine initiation. Br J Psychiatry 2012; 201: 481-5.

24 Paton C, Barnes TRE, Cavanagh M-R, Taylor D, Lelliott P. High-dose and combination antipsychotic prescribing in acute adult wards in the UK: the challenges posed by p.r.n prescribing. Br J Psychiatry 2008; 192: 435-9.

25 Tyson PJ, Mortimer AM, Wheeler JA. High-dose antipsychotic treatment in clinical practice. A review, audit and survey of consultant psychiatrist opinions. Psychiatr Bull 1999; 23: 661-4.

26 Geddes JR, Freemantle N, Harrison P, Bebbington PE. Atypical antipsychotics in the treatment of schizophrenia - systematic review and meta-regression analysis. BMJ 2000; 321: 1371-6.

27 Waddington JL, Youssef HA, Kinsella A. Mortality in schizophrenia. Antipsychotic polypharmacy and absence of adjunctive anticholinergics over the course of a 10-year prospective study. Br J Psychiatry 1998; 173: $325-9$.

28 Curkendall SM, Mo J, Glasser DB, Rose Stang $M$, Jones JK. Cardiovascular disease in patients with schizophrenia in Saskatchwan, Canada. J Clin Psychiatry 2004; 65: 715-20

29 Barnes TRE, Paton C. Role of the Prescribing Observatory for Mental Heath. Br J Psychiatry 2012; 201: 428-9. 